\title{
Erratum to: Learning Vocabularies over a Fine Quantization
}

\author{
Andrej Mikulik • Michal Perdoch • Ondřej Chum • \\ Jiří Matas
}

Published online: 1 December 2013

(C) Springer Science+Business Media New York 2013

Erratum to: Int J Comput Vis (2013) 103:163-175

DOI 10.1007/s11263-012-0600-1

Acknowledgments The authors would like to acknowledge the grant agency in the original publication. The authors were supported by the GACR P103/12/2310 project.

The online version of the original article can be found under doi:10.1007/s11263-012-0600-1.

\footnotetext{
e-mail: mikulik@cmp.felk.cvut.cz

M. Perdoch

e-mail: predom1@cmp.felk.cvut.cz

O. Chum

e-mail: chum@cmp.felk.cvut.cz

J. Matas

e-mail: matas@cmp.felk.cvut.cz
}

A. Mikulik $(\varangle) \cdot$ M. Perdoch · O. Chum · J. Matas

CMP, Department of Cybernetics, Faculty of Electrical Engineering, Czech Technical University in Prague, Prague, Czech Republic 